\title{
Retroperitoneal Hematoma after Transforaminal Selective Epidural Block: A Case Report
}

\author{
Si-Woo Lee, Yong-Woo Lee, Woo-Ram Shin, Dong-Kyu Shin \\ Department of Neurosurgery, Presbyterian Medical Center, Jeonju, Korea
}

Corresponding Author:

Woo-Ram Shin, MD, PhD

Department of Neurosurgery,

Presbyterian Medical Center, 365 Seowon-ro,

Wansan-gu, Jeonju, Jeonbuk 54987, Korea

Tel: +82-63-230-1420, Fax: +82-63-285-9573

E-mail: 1sw0215@naver.com

Received: May 25, 2016, Revised: July 13, 2016

Accepted: July 20, 2016
Transforaminal selective epidural block (TFSEB) technique is used for spinal pain management. Multiple recent reports cite TFSEB resulting in complications such as life-threatening infections, spinal fluid leaks, positional headaches, adhesive arachnoiditis, hydrocephalus, air embolism, urinary retention, allergic reactions, intravascular injections, stroke, blindness, neurological deficits/ paralysis, hematomas, seizure and death. We present herein a case of TFSEB induced retroperitoneal hematoma resolution with conservative treatment.

Key Words: Anticoagulants, Hematoma, Nerve block, Platelet aggregation inhibitors, Steroids

\section{INTRODUCTION}

TFSEB has been used to treat patients with herniated intravertebral disc, spinal stenosis, or lumbosacral radiculopathy, ${ }^{5,22)}$. It is known to reduce radicular pain by lowering inflammation of nerve roots as epidural corticosteroid injection ${ }^{7)}$.

Steroid injections at the epidural space have two approaches, interlaminar and transforaminal approaches. Compared to interlaminar approach, the use of transforaminal approach is becoming increased due to high specificity even with small amount of injection ${ }^{7)}$. The epidural space contains loose areolar connective tissue, semi-liquid fat, lymphatics, arteries, venous plexuses, and spinal nerve roots".

Although TFSEB is usually done by fluoroscopic guidance, it cannot be free from several possible complications ${ }^{5}$. Recent several studies showed there could be complications such as positional headaches (most common, 3\%), spinal fluid leaks, radiation exposure, life-threatening infections, adhesive arachnoiditis, hydrocephalus, air embolism, urinary retention, allergic reactions, intravascular injections of medication, stroke, blindness, neurological deficits/paralysis, epidural hematomas, seizure and death ${ }^{3,5,21)}$. Those complications could be occurred during needle placement or injecting medications. We present herein a case of retroperitoneal hematoma after TFSEB.

\section{CASE REPORT}

69 year old male patient presenting with right buttok and leg pain was diagnosed at a local clinic with spinal stenosis at L3-4 and L4-5, herniated interveterbral disc L3-4, and compressed right $\mathrm{L} 4$ nerve root. TFSEB was performed, and the right flank pain began 2 hours post procedure. 10 minutes later, the pain extended to the right upper quadrant. He came to the emergency department on three and half hours after TFSEB with severe pain. The physical examination was normal and showed full range of motion (ROM). There was no sign for neural compression such as numbness, weakness, bowel and bladder dysfunction, severe radicular leg pain. According to his past medical history, he had percutaneous coronary intervention (PCI) stenting 7 times from 1998 to 2009 and coronary artery bypass graft (CABG) in 2009, and he daily took two antiplatelet agents. He did not stop taking antiplatelet before TFSEB.

The abdomen and pelvis computed tomography (CT) showed large right retroperitoneal hematoma with active arterial bleeding which may indicate damaged spinal branch of segmental artery (Fig. 1). After discussion with cardiologist, antiplatelet agents were all ceased. His vital sign was stable, and all of the blood tests including hemoglobin $(13.8 \mathrm{~g} / \mathrm{dL})$ were normal. He was admitted to the intensive care unit with close monitoring.

His Hemoglobin dropped gradually from 13.8 to 9.3 for 24 hours and increased to 12.1 for 7 days. On the eighth day, the abdomen and pelvis CT showed that the amount of the retroperitoneal hematoma increased slightly from the previous state and that the internal active bleeding disappeared (Fig. 2). He moved to the general ward, and one antiplatelet agent was given again after discussing with the cardiologist. And he discharged from the hospital on the $16^{\text {th }}$ day with a healthy condition. 

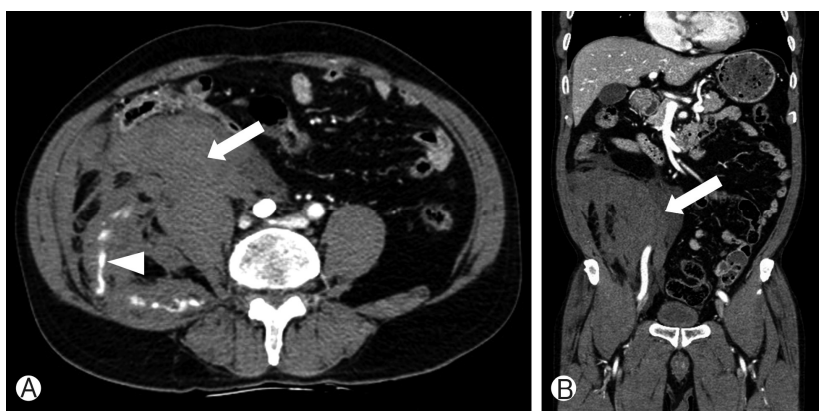

Fig. 1. Abdomen and pelvis CT 1 day post hospitalization (A) axial view, Arrow: large ill-defined heterogeneous hyperdense mass adjacent to the inner border of right psoas muscle, quadratus lumborum, and transversalis muscle, representing large right retroperitoneal hematoma. Arrow head: contrast extravasation within the mass shown indicating active arterial bleeding (B) coronal view, large right retroperitoneal hematoma.
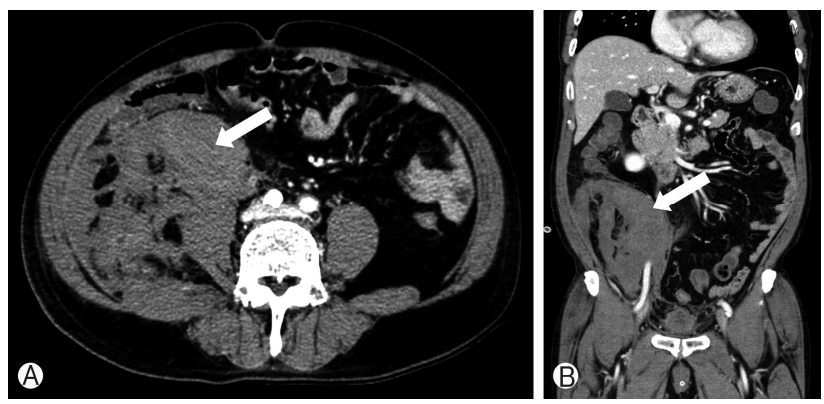

Fig. 2. Abdomen and pelvis CT 8 day post hospitalization (A), (B)) axial and coronal view, active internal bleeding sign has resolved in comparison to the image from Fig. 1.

He visited the outpatient clinic after 1 month with relieved right flank pain and right upper quadrant pain. The other antiplatelet agent was added according to the cardiologist's opinion.

He had no neurological symptom other than the abdominal pain which had a retroperitoneal location. The follow-up exams showed no active bleeding, so he underwent observation process.

\section{DISCUSSION}

The goal of TFSEB is to reduce axial and/or radicular pain by treating inflammation at nerve roots, dorsal root ganglions, anterolateral dura, posterior longitudinal ligament and intervertebral disc. Steroids and anesthetics are injected to cover nerve roots with inflammation and lateral and anterior epidural space ${ }^{3,5,7)}$. Its pain reducing mechanism can be explained by several physiologic mechanisms, which are, for examples, anti-inflammatory reaction interfering against pain-spasm cycle and suppression of phospholipase $\mathrm{A} 2$ and unmyelinated C-fiber action ${ }^{6,7,16)}$.

Intervertebral foramen is formed by superiorly and inferiorly vertebrae pedicles, anteriorly vertebral body and intervertebral disc, posteriorly articular process making zygapophyseal joint, laterally psoas muscle and fascia, and medially dural sleeve ${ }^{8)}$. Spinal nerve, dorsal root ganglion, segmental spinal artery, communicating veins between internal and external plexuses, recurrent meningeal (sinu-vertebral) nerves, and transforaminal ligaments go through the foramen ${ }^{8)}$.

31 pairs of the segmental arteries ( 8 cervical, 12 thoracic, 5 lumbar, 5 sacral, 1 coccygeal) branching off from the aorta divides into radicular artery (supplies dorsal and ventral nerve roots), radiculopial artery or radiculomedullary artery, and then posterior (paired) or anterior (single) spinal artery as they travel more distally ${ }^{17)}$.

Hematoma is pontential danger from all procedure involved with injections ${ }^{14)}$. The risk factors of hematoma are old age, female gender, liver disease, needles with large diameters, anticoagulant therapy or coagulopathy, anatomical abnormalities of the spinal cord and vertebral column, and difficult/traumatic needle placement ${ }^{10,23)}$.

There have been debates about need for discontinuation of anticoagulant or antiplatelet therapy which is done for cerebral infarction, angina, or pulmonary embolism.

Horlocker et al. reported that patients with heparin anticoagulants are at 10 times bigger bleeding risk than those without them ${ }^{13)}$, and Layton et al. said that use of multiple anticoagulants evidently increased the bleeding risk ${ }^{19)}$. Hsu DT et al. suggests to stop warfarin 5 days before the procedure and restart after 3 days post procedure ${ }^{14)}$. American Society of Regional Anesthesia (ASRA) said that warfarin should be stopped 4 to 5 days before spinal procedures and that international normalized ratio (INR) and vitamin $\mathrm{K}$-dependent factor should be within normal ranges at the time of the procedure. It also said thienopyridine drugs such as clopidogrel and ticlopidine should be stopped for 7 to 14 days and low-molecular weight heparin (LMWH) should be stopped at least 12 hours before procedures ${ }^{12)}$. Another study showed that aspirin and non-steroidal anti-inflammatory drugs (NSAID) were not related to complications from spinal procedure ${ }^{11,12)}$.

However, Manchikanti said bleeding complication was still similar whether the use of anticoagulation was kept or stopped, and thromboembolic event increased by 17 times from the discontinuation of the drugs ${ }^{20)}$.

The optimal target anatomical location of the transforaminal epidural steroid injection has been known to be at the posterior surface of the vertebral body adjacent to the caudal border of the pedicle immediately superior to the target nerve ${ }^{5)}$. Usually if the needle is inserted deeper than the optimal target location, terminal branch of the segmental artery located postero-laterally to the vertebral body may be damaged and induce retroperitoneal hematoma.

If there is a sign for this kind of nerve injury, location of hematoma should be evaluated by MRI or CT, and evacuation should be done within 24 hours to minimize nerve injury ${ }^{18)}$. For an accurate procedure, TFSEB is usually done through fluoroscopic guidance, but it cannot be free from the several possible complications mentioned above ${ }^{5)}$.

James et al. proposed several factors to be considered for safe procedure during TFSEB. Use of non-particulate steroid 
may lower catastrophic neurovascular complication. Particulate steroid should not be used. Before injecting steroid or anesthetics, contrast medium injection under real-time fluoroscopy should be done. A needle should be firmly located once with extension tubing, which makes no need to relocate the needle. One must make a decision for which way to approach to a target considering risks and benefits for all procedures ${ }^{21)}$.

\section{CONCLUSION}

For successful TFSEB, one should understand thoroughly the anatomy and pay attention to possible risks even though it has been reported that complications are quite lo. It may be better to discontinue the drugs if possible. If use of the drugs must be kept due to patients' underlying diseases, other treatment options instead of TFSEB can be considered.

\section{REFERENCES}

1. Aprill CN, Melfi RS: Paraplegia after lumbosacral nerve root block: report of three cases. Spine J 4(3):368-9, 2004

2. Aveline C, Bonnet F: Delayed retroperitoneal haematoma after failed lumbar plexus block. Br J Anaesth 93:589-91, 2004

3. Bicket MC, Chakravarthy K, Chang D, Cohen SP: Epidural steroid injections: an updated review on recent trends in safety and complications. Pain Manag 5(2):129-46, 2015

4. Botwin KP, Gruber RD, Bouchlas CG, Torres-Ramos FM, Freeman TL, Slaten TK: Complications of fluoroscopically guided transforaminal lumbar epidural injections. Arch Phys Med Rehabil 81(8):1045-50, 2000

5. Bradly S, Lyle W, Srinivas M, Matt B: Complications and pitfalls of lumbar interlaminar and transforaminal epidural injections. Curr Rev Musculoskelet Med 1(3-4):212-22, 2008

6. Derby R, Kine G, Saal JA, Reynoids J, Goldthwaite N, White $\mathrm{AH}$, et al: Response to steroid and duration of radicular pain as predictors of surgical outcome. Spine (Phila Pa 1976) 17(16 Suppl):176-83, 1992

7. Desai MJ, Dua S: Perineural hematoma following lumbar transforaminal steroid injection causing acute-on-chronic lumbar radiculopathy: a case report. Pain Pract 14(3):271-7, 2014

8. Gilchrist RV, Slipman CW, Bhagia SM: Anatomy of the intervertebral foramen. Pain Physician 5(4):372-8, 2002

9. Hogan $\mathrm{QH}$ : Epidural anatomy examined by crymicrotome section. Influence of age, vertebral level, and disease. Rge Anesth 21(5): 39-406, 1996

10. Horlocker TT: Regional anaesthesia in the patient receiving antithrombotic and antiplatelet therapy. Br J Anaesth 107(Suppl 1):i96-106, 2011
11. Horlocker TT, Bajwa ZH, Ashrff Z, Khan S, Wilson JL, Sami $\mathrm{N}$, et al. Risk assessment of hemorrhagic complications associated with nonsteroidal antiinfalmmatory medications in ambulatory pain clinic patients undergoing epidural steroid injection. Anesth Analg 95(6):1691-7, 2002

12. Horlocker TT, Wedel DJ, Benzon H, Brown DL, Enneking FK, Heit JA, et al: Regional anesthesia in the anticoagulated patient: defining the risks (the second ASRA consensus conference on neuraxial anesthesia and anticoagulation). Rge Anesth Pain Med 28(3):172-97, 2003

13. Horlocker TT, Wedel DJ, Rowlingson JC, Enneking FK, Kopp SL, Benzon HT, et al: Regional anesthesia in the patient receiving antithrombotic or thrombolytic therapy: American Society of Regional Anesthesia and Pain Medicine evidence -based guidelines (third edition). Reg Anesth Pain Med 35(1):64-101, 2010

14. Hsu DT: Delayed retroperitoneal haematoma after failed lumbar plexus block. Br J Anaesth 94:395-6, 2005

15. Huston CW, Slipman CW, Garvin C: Complications and side effects of cervical and lumbosacral selective nerve root injections. Arch Phys Rehabil 86(2):277-83, 2005

16. Johansson A, Hao J, Sjölund B: Local corticosteroid application blocks transmission in normal nociceptive C-fibers. Acta Anaesthesiol Scand 34(5):335-8, 1990

17. Krauss WE: Vascular anatomy of the spinal cord. Neurosurg Clin N Am 10(1):9-15, 1999

18. Lawton MT, Porter RW, Heiseman JE, Jacobowitz R, Sonntag VK, Dickman CA: Surgical management of spinal epidural hematoma: relationship between surgical timing and neurological outcome. J Neurosurg 83(1):1-7, 1995

19. Layton KF, Kallmes DF, Horlocker TT: Recommendations for anticoagulated patients undergoing image-guided spinal procedures. AJNR Am J Neuroradiol 27(3):468-70, 2006

20. Manchikanti L, Benyamin RM, Swicegood JR, Falco FJ, Datta S, Pampati V, et al: Assessment of practice patterns of perioperative management of antiplatelet and anticoagulant therapy in interventional pain management. Pain Physician 15(6):E95568, 2012

21. Rathmell JP, Benzon HT, Dreyfuss P, Huntoon M, Wallace M, Baker R, et al: Safeguards to prevent neurologic complications after epidural steroid injections: consensus opinions from a multidisciplinary working group and national organizations. Anesthesiology 122(5):974-84, 2015

22. Vad V, Bhat A, Lutz G, Cammisa F: Transforaminal epidural steroid injections in lumbosacral radiculopathy: a prospective randomized study. Spine 27:11-16, 2002

23. Windsor RE, Storm S, Sugar R: Prevention and management of complications resulting from common spinal injections. Pain Physician 6(4):473-83, 2003 